\title{
APPLICATIONS OF THE ASYMPTOTIC LARGE SIEVE
}

\author{
by
}

Brian Conrey

\begin{abstract}
In this note we describe some results of Conrey, Iwaniec, and Soundararajan. The details will appear elsewhere.

Résumé. - Dans cette note, nous présentons des résultats de Conrey, Iwaniec et Soundararajan. Les détails seront publiés ultérieurement.
\end{abstract}

\section{Introduction}

The large sieve inequality, in its multiplicative form [H-B1], asserts that for any sequence of complex numbers $a_{n}$,

$$
\mathcal{S}(\vec{a}, Q):=\sum_{q \leq Q} \frac{q}{\phi(q)} \sum_{\chi \bmod q}^{*}\left|\sum_{n=1}^{N} a_{n} \chi(n)\right|^{2} \leq\left(Q^{2}+N\right) \sum_{n=1}^{N}\left|a_{n}\right|^{2} .
$$

Many applications have followed from this basic tool, especially, in this form, applications having to do with primes in arithmetic progressions, or to do with statistical averages of Dirichlet L-functions. Generalizations of this basic tool have been given where the characters $\chi$ are replaced by the coefficients of L-functions from a family. Thus, there are versions which involve only the real characters (see [H-B4], as well as versions with Fourier coefficients of modular forms (see [DI], [Iwa1], and [Iwa2]) and many others. The large sieves reveal an almost orthogonality among the sets of coefficients.

For certain applications, it is desirable to have an asymptotic formula in place of the inequality above. We have developed such an asymptotic formula, for certain sequences, and even have formulas for

$$
\mathcal{S}(\vec{a}, Q):=\sum_{q \leq Q} \frac{q}{\phi(q)} \sum_{\chi \bmod q}^{*}|L(1 / 2, \chi)|^{2}\left|\sum_{n=1}^{N} a_{n} \chi(n)\right|^{2}
$$

2000 Mathematics Subject Classification. - 11M06, 11M26.

Key words and phrases. - Large sieve, Dirichlet L-functions, Generalized Riemann Hypothesis, moments of L-functions. 
for L-functions of degrees 1, 2, and 3 and for

$$
\mathcal{S}(\vec{a}, Q):=\sum_{q \leq Q} \frac{q}{\phi(q)} \sum_{\chi \bmod q}^{*}|L(1 / 2, \chi)|^{2 k}\left|\sum_{n=1}^{N} a_{n} \chi(n)\right|^{2}
$$

provided that $k$ times the degree of $\mathrm{L}$ is not too large.

As a sample application, we can show that, assuming the generalized Riemann Hypothesis, there are $L$-functions which have zeros spaced closer than 0.366 times the average spacing. Another application is to critical zeros of a family of twists of a fixed L-function. Let $L(s)$ be an L-function. We consider the collection

$$
\{L(s, \chi)\}
$$

where $\chi$ ranges over all primitive characters $\chi$ modulo $q$ with $q \leq Q$. We consider all of the zeros of all of these $L$-functions up to a height $\log Q$. If $L$ is a degree one L-function, then at least $55 \%$ of all of these zeros are on the $1 / 2$-line. If $L$ is degree 2 , then at least $35 \%$ are on the critical line, and if $L$ is degree 3 then at least one-half of one percent of the zeros are on the critical line.

A third application is to moments of Dirichlet L-functions. Here the best result is for the sixth moment of Dirichlet L-functions. We prove a formula, which contains the full ninth degree polynomial polynomial, and which agrees perfectly with the conjectures motivated by random matrix theory.

\section{The basic idea}

The essence of $S(\vec{a}, Q)$ is $\Delta$ which is defined through

$$
S(\vec{a}, Q)=\sum_{m, n \leq X} a_{m} b_{n} \Delta(m, n)
$$

thus

$$
\Delta(m, n):=\sum_{q} \frac{W(q / Q)}{\phi(q)} \sum_{\chi \bmod q}^{*} \chi(m) \overline{\chi(n)} .
$$

Here are some basic lemmas to get us started analyzing this $\Delta$-symbol.

Lemma 2.1. - If $(m n, q)=1$, then

$$
\sum_{\chi \bmod q}^{*} \chi(m) \overline{\chi(n)}=\sum_{\substack{d|q \\ d|(m-n)}} \phi(d) \mu(q / d) .
$$

Applying Lemma 2.1 we find that

$$
\Delta(m, n)=\sum_{\substack{(c d, m n)=1 \\ d \mid m-n}} \frac{W(c d / Q) \mu(c) \phi(d)}{\phi(c d)} .
$$


Lemma 2.2. - We have

$$
\frac{\phi(d)}{\phi(c d)}=\frac{1}{\phi(c)} \sum_{\substack{a|c \\ a| d}} \frac{\mu(a)}{a}
$$

Thus,

$$
\Delta(m, n)=\sum_{\substack{(a c d, m n)=1 \\ a d \mid(m-n)}} \frac{W\left(a^{2} c d / Q\right) \mu(a) \mu(a c)}{a \phi(a c)} .
$$

Now we separate the diagonal terms from the non-diagonal ones.

Proposition 2.3. - We have

$$
\Delta(m, m)=\hat{W}(1) Q \frac{\phi(m)}{m} \prod_{p}\left(1-\frac{1}{p^{2}}-\frac{1}{p^{3}}\right) \prod_{p \mid m}\left(1-\frac{1}{p^{2}}-\frac{1}{p^{3}}\right)^{-1}+O_{\epsilon}\left((Q m)^{\epsilon}\right)
$$

Proof. - We have

$$
\begin{aligned}
\Delta(m, m) & =\sum_{(a c d, m)=1} \frac{\mu(a) \mu(a c)}{a \phi(a c)} W\left(\frac{a^{2} c d}{Q}\right) \\
& =\frac{1}{2 \pi i} \int_{(2)} Q^{s} \hat{W}(s) \zeta(s) \prod_{p \mid m}\left(1-\frac{1}{p^{s}}\right) \sum_{(a c, m)=1} \frac{\mu(a) \mu(a c)}{a^{1+2 s} c^{s} \phi(a c)} d s .
\end{aligned}
$$

The sums over $a$ and $c$ are absolutely convergent for $\sigma>0$ and $\hat{W}(s)$ is of rapid decay in the vertical direction. Let $\epsilon>0$. We shift the path of integration to the $\epsilon$-line and pick up the residue from the pole of $\zeta(s)$ at $s=1$. Thus

$$
\Delta(m, m)=\hat{W}(1) Q \frac{\phi(m)}{m} \sum_{(a c, m)=1} \frac{\mu(a) \mu(a c)}{a^{3} c \phi(a c)}+O\left((Q m)^{\epsilon}\right) .
$$

The sum over $a$ and $c$ in the main term is

$$
=\prod_{p \nmid m}\left(1+\frac{1}{p^{3}(p-1)}-\frac{1}{p(p-1)}\right)=\prod_{p \nmid m}\left(1-\frac{1}{p^{2}}-\frac{1}{p^{3}}\right) .
$$

Now we shall assume that $m \neq n$. We introduce a parameter $C$ and split the sum over $c$ in $\Delta$ so that we have $\Delta(m, n)=L(m, n)+U(m, n)$ where

$$
L(m, n)=\sum_{\substack{(a c d, m n)=1 \\ a d \mid m-n, c \leq C}} \frac{W\left(a^{2} c d / Q\right) \mu(a) \mu(a c)}{a \phi(a c)}
$$

and

$$
U(m, n)=\sum_{\substack{(a c d, m n)=1 \\ a d \mid m-n, c>C}} \frac{W\left(a^{2} c d / Q\right) \mu(a) \mu(a c)}{a \phi(a c)}
$$


The term $U(m, n)$ is relatively easy to analyze. Basically we detect the congruence $m \equiv$ $n$ mod $a d$ by going to characters modulo $a d$. Since $c$ is large and $a c d \approx Q$ it must be the case that $a d$ is small and so it is not too expensive to sum over all of the characters modulo $a d$. For the term $L(m, n)$ with smaller $c$ and larger ad we write $|m-n|=$ ade where $e$ is called the complementary modulus and we then, after elimination of $d$ from the rest of the sum, we have $m \equiv n \bmod a e$ and detect this congruence by characters modulo ae. The difficulty in doing this is largely technical; to eliminate the variable $d$ everywhere by replacing it by $|m-n| /$ ae is complicated! In carrying out this procedure for specific sequences $a_{n}$ one often encounters main terms arising from the principal characters modulo ad in $U$ and from the principal characters modulo ae in $L$. The two largest of these main terms cancel; the real offdiagonal contribution comes from a secondary main term in the principal characters modulo ae of $L$. This term combines with the diagonal to form the final main term.

In the next two sections we give further examples of precise theorems we can prove.

\section{The analogue of the Balasubramanian, Conrey, Heath-Brown conjecture}

Theorem 3.1. - Suppose that $W$ is smooth and supported on $[1,2]$. Then

$$
\begin{aligned}
& \sum_{q} W\left(\frac{q}{Q}\right) \sum_{\chi \bmod q}^{b}|L(1 / 2, \chi)|^{2} \chi(h) \bar{\chi}(k)=\operatorname{Res}_{s=0}\left(\tilde{W}(s+2) \pi^{-s} \frac{\Gamma\left(\frac{s+1 / 2}{2}\right)^{2}}{\Gamma\left(\frac{1}{4}\right)^{2}} Q^{2+s} \frac{\zeta(1+2 s)}{s}\right. \\
& \left.\times \frac{\phi(h k)}{h k} \frac{(h, k)^{1+2 s}}{(h k)^{1 / 2+s}} \prod_{p \nmid h k}\left(1-\frac{1}{p^{2+2 s}}-\frac{2}{p^{2}}+\frac{2}{p^{3+2 s}}+\frac{1}{p^{4}}-\frac{1}{p^{5+2 s}}\right)\right)+\mathcal{E}_{h, k}
\end{aligned}
$$

where

$$
\sum_{h, k \leq N} \lambda_{h} \overline{\lambda_{k}} \mathcal{E}_{h, k} \ll\left(Q^{2-\epsilon}+(Q N)^{1+\epsilon}\right) \sum_{h \leq N}\left|(\lambda * \lambda)_{h}\right|^{2} .
$$

Here $\lambda * \lambda$ is the Dirichlet convolution of the sequence $\lambda$ with itself. Note that for a sequence $\lambda_{h} \ll h^{\epsilon-1 / 2}$ the main term above is likely to be $\gg Q^{2}$ so that we have an asymptotic formula provided that $N \ll Q^{1-\epsilon}$.

The above is the analogue of a conjecture of Balasubramanian, Conrey, and Heath-Brown [BCH-B] who proved that

$\int_{0}^{T}|\zeta(1 / 2+i t)|^{2}\left|\sum_{n=1}^{N} \lambda_{n} n^{-i t}\right|^{2} d t=T \sum_{h, k \leq N} \lambda_{h} \overline{\lambda_{k}}\left(\frac{(h, k)}{\sqrt{h k}}\left(\log \frac{T(h, k)^{2}}{2 \pi h k}+2 \gamma-1\right)+\mathcal{E}(h, k)\right)$

where

$$
\sum_{h, k \leq N} \lambda_{h} \overline{\lambda_{k}} \mathcal{E}_{h, k} \ll\left(T^{1-\epsilon}+T^{1 / 2} N\right) \sum_{n \leq N}\left|\lambda_{n}\right|^{2} .
$$

This formula gives an asymptotic formula provided that $N \ll T^{1 / 2-\epsilon}$. The authors conjecture that the asymptotic formula actually holds provided that $N \ll T^{1-\epsilon}$ (a conjecture which is equivalent to the Lindelöf hypothesis). 


\section{The sixth moment}

Understanding moments of families of $L$-functions has long been an important subject with many number theoretic applications. It is only in the last ten years that an understanding of the finer structure of moments has begun to emerge. The new vision began with the work of Keating and Snaith, who realized that the distribution of the values of an L-function, or family of L-functions, can be modeled by characteristic polynomials from classical compact groups, and to Katz and Sarnak for their realization that families of L-functions have symmetry types associated with them that reveal which of the classical groups to use to model the family. See [KaSa], [KS1], [KS2], and [CF].

Prior to these works, Conrey and Ghosh predicted, on number theoretic grounds, that

$$
\int_{0}^{T}|\zeta(1 / 2+i t)|^{6} d t \sim 42 \prod_{p}\left(1-\frac{1}{p}\right)^{4}\left(1+\frac{4}{p}+\frac{1}{p^{2}}\right) T \frac{\log ^{9} T}{9 !} .
$$

The conjecture of Keating and Snaith agrees with this. Numerically, however, this conjecture is untestable. For example,

$$
\int_{0}^{2350000}|\zeta(1 / 2+i t)|^{6} d t=3317496016044.9=3.3 \times 10^{12}
$$

whereas

$$
42 \prod_{p}\left(1-\frac{1}{p}\right)^{4}\left(1+\frac{4}{p}+\frac{1}{p^{2}}\right) \times 2350000 \times \frac{(\log 2350000)^{9}}{9 !}=4.22 \times 10^{11}
$$

is nowhere near the prediction. This situation has been rectified by the conjectures of [CFKRS] which assert, for example, that for any $\epsilon>0$,

$$
\int_{0}^{T}|\zeta(1 / 2+i t)|^{6} d t=\int_{0}^{T} P_{3}\left(\log \frac{t}{2 \pi}\right) d t+O\left(T^{1 / 2+\epsilon}\right)
$$

where $P_{3}$ is a polynomial of degree 9 whose exact coefficients are specified as complicated infinite products and series over primes, but whose approximate coefficients are

$$
\begin{aligned}
& P_{3}(x)=0.000005708 x^{9}+0.0004050 x^{8}+0.01107 x^{7}+0.1484 x^{6} \\
& \quad+1.0459 x^{5}+3.9843 x^{4}+8.6073 x^{3}+10.2743 x^{2}+6.5939 x+0.9165 .
\end{aligned}
$$

For this polynomial, we have

$$
\int_{0}^{2350000} P_{3}\left(\log \frac{t}{2 \pi}\right) d t=3317437762612.4
$$

which agrees well with the numerics.

A proof of an asymptotic formula for the sixth moment of $\zeta(s)$ on the citical line is completely out of reach of today's technology. However, we have proved an analogous formula for Dirichlet $L$-functions suitably averaged. Our formula agrees exactly with the conjecture of [CFKRS] and so provides, we hope, a new glimpse into the mechanics of moments. 
The precise statement of our theorem on the sixth moment of Dirichlet L-functions is a little complicated to state and requires some set up. We invite the reader to look at the end of the paper for a version of the theorem which shows the first main term in the asymptotic formula. Let $\chi \bmod q$ be an even, primitive Dirichlet character and let

$$
L(s, \chi)=\sum_{n=1}^{\infty} \frac{\chi(n)}{n^{s}}
$$

be its associated $L$-function. Such an $L$-function has an Euler product: $L(s, \chi)=\prod_{p}(1-$ $\left.\chi(p) / p^{s}\right)^{-1}$ and a functional equation

$$
\Lambda(s, \chi):=(q / \pi)^{(s-1 / 2) / 2} \Gamma(s / 2) L(s, \chi)=\epsilon_{\chi} \Lambda(1-s, \bar{\chi})
$$

where $\epsilon_{\chi}$ is a complex number of absolute value 1 . We prove an asymptotic formula, with a power savings, for a suitable average of the sixth power of the absolute value of these primitive Dirichlet $L$-functions near the critical point $1 / 2$.

An upper bound for such an average follows from the large sieve inequality. Huxley used this to prove that

$$
\sum_{q \leq Q} \sum_{\chi \bmod q}^{*}|L(1 / 2, \chi)|^{6} \ll Q^{2} \log ^{9} Q
$$

and

$$
\sum_{q \leq Q} \sum_{\chi \bmod q}^{*}|L(1 / 2, \chi)|^{8} \ll Q^{2} \log ^{16} Q .
$$

Here is a statement of those conjectures and the theorem of this paper. Let $A$ and $B$ be sets of complex numbers with equal cardinality $|A|=|B|=K$. Suppose that $|\Re \alpha|,|\Re \beta| \leq 1 / 4$ for $\alpha \in A, \beta \in B$. These are the "shifts". Let

$$
\begin{aligned}
\Lambda_{A, B}(\chi) & :=\prod_{\alpha \in A} \Lambda(1 / 2+\alpha, \chi) \prod_{\beta \in B} \Lambda(1 / 2+\beta, \bar{\chi}) \\
& =\left(\frac{q}{\pi}\right)^{\delta_{A, B}} G_{A, B} \mathcal{L}_{A, B}(\chi)
\end{aligned}
$$

where

$$
\begin{aligned}
\mathcal{L}_{A, B}(\chi) & :=\prod_{\alpha \in A} L(1 / 2+\alpha, \chi) \prod_{\beta \in B} L(1 / 2+\beta, \bar{\chi}), \\
G_{A, B} & :=\prod_{\alpha \in A} \Gamma\left(\frac{1 / 2+\alpha}{2}\right) \prod_{\beta \in B} \Gamma\left(\frac{1 / 2+\beta}{2}\right)
\end{aligned}
$$

and

$$
\delta_{A, B}=\frac{1}{2}\left(\sum_{\alpha \in A} \alpha+\sum_{\beta \in B} \beta\right) .
$$


Further, let

$$
\mathcal{Z}(A, B):=\prod_{\substack{\alpha \in A \\ \beta \in B}} \zeta(1+\alpha+\beta)
$$

and

$$
\mathcal{A}(A, B):=\prod_{p} \mathcal{B}_{p}(A, B) \mathcal{Z}_{p}(A, B)^{-1}
$$

with

$$
\mathcal{Z}_{p}(A, B):=\prod_{\substack{\alpha \in A \\ \beta \in B}} \zeta_{p}(1+\alpha+\beta)
$$

and

$$
\zeta_{p}(x)=\left(1-\frac{1}{p^{x}}\right)^{-1}
$$

also

$$
\mathcal{B}_{p}(A, B):=\int_{0}^{1} \prod_{\alpha \in A} z_{p, \theta}(1 / 2+\alpha) \prod_{\beta \in B} z_{p,-\theta}(1 / 2+\beta) d \theta
$$

with $z_{p, \theta}(x)=1 /\left(1-e(\theta) / p^{x}\right)$. The conditions on the real parts of elements of $A$ and $B$ ensure that the Euler product for $\mathcal{A}$ converges absolutely. Let $\mathcal{B}_{q}=\prod_{p \mid q} \mathcal{B}_{p}$. Let

$$
\mathcal{Q}_{A, B}(q):=\sum_{\substack{S \subset A \\ T \subset B \\|S|=|T|}} \mathcal{Q}(\bar{S} \cup(-T), \bar{T} \cup(-S) ; q)
$$

where $\bar{S}$ denotes the complement of $S$ in $A$, and by the set $-S$ we mean $\{-s: s \in S\}$ and, for any sets $X$ and $Y$,

$$
\mathcal{Q}(X, Y ; q)=\left(\frac{q}{\pi}\right)^{\delta_{X, Y}} G_{X, Y} \frac{\mathcal{A \mathcal { Z }}}{\mathcal{B}_{q}}(X, Y) .
$$

For example, if $A=\left\{\alpha_{1}, \alpha_{2}, \alpha_{3}\right\}$ and $B=\left\{\beta_{1}, \beta_{2}, \beta_{3}\right\}$, then

$$
\begin{gathered}
\mathcal{Q}_{A, B}(q)=\mathcal{Q}\left(\left\{\alpha_{1}, \alpha_{2}, \alpha_{3}\right\},\left\{\beta_{1}, \beta_{2}, \beta_{3}\right\}, q\right)+\mathcal{Q}\left(\left\{-\beta_{1}, \alpha_{2}, \alpha_{3}\right\},\left\{-\alpha_{1}, \beta_{2}, \beta_{3}\right\}, q\right) \\
+\cdots+\mathcal{Q}\left(\left\{-\beta_{1},-\beta_{2},-\beta_{3}\right\},\left\{-\alpha_{1},-\alpha_{2},-\alpha_{3}\right\}, q\right),
\end{gathered}
$$

is a sum of 8 terms; the first summand corresponds to $S=T=\phi$ so that $X=A$ and $Y=B$; the second summand corresponds to $S=\left\{\alpha_{1}\right\}$ and $T=\left\{\beta_{1}\right\}$, and so on. In general, $\mathcal{Q}_{A, B}(q)$ will have $\left(\begin{array}{c}2 K \\ K\end{array}\right)$ summands.

Now we state the conjecture.

Conjecture 4.1. - [CFKRS] Assuming that the "shifts" $\alpha \in A, \beta \in B$ satisfy $|\Re \alpha|,|\Re \beta| \leq$ $1 / 4$, and $\Im \alpha, \Im \beta \ll q^{1-\epsilon}$, we conjecture that

$$
\sum_{\chi \bmod q}^{b} \Lambda_{A, B}(\chi)=\sum_{\chi \bmod q}^{b} \mathcal{Q}_{A, B}(q)\left(1+O\left(q^{-1 / 2+\epsilon}\right)\right)
$$


where $\sum^{b}$ denotes a sum over even primitive characters.

When $|A|=|B|=3$ and all of the shifts are 0 , the conjecture implies that, as $q \rightarrow \infty$ with $q$ not congruent to 2 modulo 4 , we have

\section{Conjecture 4.2. - [CFKRS]}

$$
\frac{1}{\phi^{b}(q)} \sum_{\chi \bmod q}^{b}\left|L\left(\frac{1}{2}, \chi\right)\right|^{6} \sim 42 a_{3} \prod_{p \mid q} \frac{\left(1-\frac{1}{p}\right)^{5}}{\left(1+\frac{4}{p}+\frac{1}{p^{2}}\right)} \frac{\log ^{9} q}{9 !} .
$$

where $\phi^{b}(q)$ is the number of even primitive characters modulo $q$ and

$$
a_{3}=\prod_{p}\left(1-\frac{1}{p}\right)^{4}\left(1+\frac{4}{p}+\frac{1}{p^{2}}\right)
$$

Note that $a_{3}$ is the constant that appears in the conjecture for the sixth moment of $\zeta$. The ' 42 ' here played an important role in the discovery by Keating and Snaith that moments of $L$-functions could be modeled by moments of characteristic polynomials, see Beineke and Hughes [BeHu] for an account of this story.

For a set $A$ it is convenient to let $A_{t}$ be the set of translates

$$
A_{t}=\{\alpha+t: \alpha \in A\}
$$

Note that $\left(A_{s_{1}}\right)_{s_{2}}=A_{s_{1}+s_{2}}$.

Theorem 4.3. - Suppose that $|A|=|B|=3$ and that $\alpha, \beta \ll 1 / \log Q$, for $\alpha \in A, \beta \in B$. Suppose that $\Psi$ is smooth on $[1,2]$ and $\Phi(t)$ is an entire function of $t$ which decays rapidly as $|t| \rightarrow \infty$ in any fixed horizontal strip. Then

$$
\begin{aligned}
& \sum_{q} \Psi\left(\frac{q}{Q}\right) \int_{-\infty}^{\infty} \Phi(t) \sum_{\chi}^{b} \Lambda_{A_{i t}, B_{-i t}}(\chi) d t \\
& =\sum_{q} \Psi\left(\frac{q}{Q}\right) \int_{-\infty}^{\infty} \Phi(t) \phi^{b}(q) \mathcal{Q}_{A_{i t}, B_{-i t}}(q) d t+O\left(Q^{7 / 4+\epsilon}\right) .
\end{aligned}
$$

We could equally well prove a theorem for odd primitive characters. The answer would be similar with just the Gamma-factors changed slightly to reflect the difference in the functional equation for odd primitive Dirichlet $L$-functions. When the shifts are all 0 , this difference disappears, in the leading order main term. 
Corollary 4.4. - We have

$$
\begin{aligned}
& \sum_{q} \sum_{\chi \bmod q}^{*} \Psi(q / Q) \int_{-\infty}^{\infty} \Phi(t)|L(1 / 2+i t, \chi)|^{6} d t \\
& \quad \sim 42 a_{3} \sum_{q} \Psi\left(\frac{q}{Q}\right) \prod_{p \mid q} \frac{\left(1-\frac{1}{p}\right)^{5}}{\left(1+\frac{4}{p}+\frac{1}{p^{2}}\right)} \phi^{b}(q) \frac{\log ^{9} q}{9 !} \int_{-\infty}^{\infty} \Phi(t)|\Gamma((1 / 2+i t) / 2)|^{6} d t \\
& \quad \sim 42 a_{3}(\mathcal{L}) Q^{2} \frac{\log ^{9} Q}{9 !} \int_{0}^{\infty} \Psi(x) x d x \int_{-\infty}^{\infty} \Phi(t)|\Gamma((1 / 2+i y) / 2)|^{6} d t
\end{aligned}
$$

where

$$
a_{3}(\mathcal{L})=\prod_{p}\left(1-\frac{1}{p}\right)^{5}\left(1+\frac{5}{p}-\frac{5}{p^{2}}+\frac{14}{p^{3}}-\frac{15}{p^{4}}+\frac{5}{p^{5}}+\frac{4}{p^{6}}-\frac{4}{p^{7}}+\frac{1}{p^{8}}\right) .
$$

Thus, our theorem asserts that Conjectures 4.1 and 4.2 are true (but with a weaker error term) on average over $q$ and a mild average over $y$.

\section{Conclusion}

The asymptotic large sieve is potentially a very useful tool with many applications. In its initial incarnations, the proofs are fairly difficult, especially when complex main terms have to be unearthed. The authors are seeking good ways to present these new ideas, ways that will simplify some of these complications.

\section{References}

[BCH-B] Balasubramanian, R.; Conrey, J. B.; Heath-Brown, D. R. Asymptotic mean square of the product of the Riemann zeta-function and a Dirichlet polynomial. J. Reine Angew. Math. 357 (1985), 161-181.

[BeHu] Beineke, J. and Hughes, C. Great moments of the Riemann zeta function, in Biscuits of number theory. Edited by Arthur T. Benjamin and Ezra Brown. The Dolciani Mathematical Expositions, 34. Mathematical Association of America, Washington, DC, 2009. xiv +311 pp.

[BD] Bombieri, E.; Davenport, H. Some inequalities involving trigonometrical polynomials. Ann. Scuola Norm. Sup. Pisa (3) 23 (1969), 223-241.

[Con] Conrey, J. B. The mean-square of Dirichlet $L$-functions, arXiv:0708.2699.

[CF] Conrey, J. B.; Farmer, D. W. Mean values of $L$-functions and symmetry. Internat. Math. Res. Notices 2000, no. 17, 883-908.

[CFKRS] Conrey, J. B.; Farmer, D. W.; Keating, J. P.; Rubinstein, M. O.; Snaith, N. C. Integral moments of $L$-functions. Proc. London Math. Soc. (3) 91 (2005), no. 1, 33-104.

[CG] Conrey, J. B.; Ghosh, A. A conjecture for the sixth power moment of the Riemann zeta-function. Internat. Math. Res. Notices 1998, no. 15, 775-780.

[CGo] Conrey, J. B. ; Gonek, S. M. High moments of the Riemann zeta-function. Duke Math. J. 107 (2001), no. 3, 577-604.

[CIS] Conrey, J. B., Iwaniec, H., Soundararajan, K. The asymptotic large sieve. In preparation. 
[DI] Deshouillers, J.-M.; Iwaniec, H. Kloosterman sums and Fourier coefficients of cusp forms. Invent. Math. $70(1982 / 83)$, no. 2, 219-288.

[H-B1] Heath-Brown, D. R. The fourth power moment of the Riemann zeta function. Proc. London Math. Soc. (3) 38 (1979), no. 3, 385-422.

[H-B2] Heath-Brown, D. R. An asymptotic series for the mean value of Dirichlet $L$-functions. Comment. Math. Helv. 56 (1981), no. 1, 148-161.

[H-B3] Heath-Brown, D. R. The fourth power mean of Dirichlet's L-functions. Analysis 1 (1981), no. $1,25-32$.

[H-B4] Heath-Brown, D. R. A mean value estimate for real character sums. Acta Arith. 72 (1995), no. $3,235-275$.

[Hux] Huxley, M. N. The large sieve inequality for algebraic number fields. II. Means of moments of Hecke zeta-functions. Proc. London Math. Soc. (3) 21 (1970), 108-128.

[Iwa1] Iwaniec, Henryk Introduction to the spectral theory of automorphic forms. Biblioteca de la Revista Matematica Iberoamericana. Revista Matematica Iberoamericana, Madrid, 1995.

[Iwa2] Iwaniec, Henryk Topics in classical automorphic forms. Graduate Studies in Mathematics, 17. American Mathematical Society, Providence, RI, 1997.

[KaSa] Katz, Nicholas M.; Sarnak, Peter Random matrices, Frobenius eigenvalues, and monodromy. American Mathematical Society Colloquium Publications, 45. American Mathematical Society, Providence, RI, 1999.

[KS1] Keating, J. P.; Snaith, N. C. Random matrix theory and $\zeta(1 / 2+i t)$. Comm. Math. Phys. 214 (2000), no. 1, 57-89.

[KS2] Keating, J. P.; Snaith, N. C. Random matrix theory and $L$-functions at $s=1 / 2$. Comm. Math. Phys. 214 (2000), no. 1, 91-110.

[Sou] Soundararajan, K. The fourth moment of Dirichlet $L$-functions, Analytic number theory, 239246, Clay Math. Proc., 7, Amer. Math. Soc., Providence, RI, 2007; arxiv math.NT/0507150.

[Sou1] Soundararajan, K. Moments of the Riemann zeta-function, Ann. of Math. (2) 170 (2009), no. 2, 981-993.; arxiv math.NT/0612106.

[You] Young, Matthew. The fourth moment of Dirichlet $L$-functions, to appear, Ann. of Math.;arxiv math.NT/0610335.

Lundi 31 janvier 2011

Brian Conrey, American Institute of Mathematics, 360 Portage Ave Palo Alto, CA 94306-2244 Department of Mathematics University Walk, Clifton, Bristol BS8 1TW, U.K.

E-mail : conrey@aimath.org 